\section{Refractory thrombocytopenia in a severe COVID-19 patient}

\author{
Mirko Di Capua, Alessio Conca, \\ Simone Testa, Maria Mascolo, \\ Stefano Paglia
}

Emergency Department, ASST Lodi, Italy

\begin{abstract}
Severe acute respiratory syndromerelated coronavirus 2 (SARS-CoV-2) infection has spread worldwide. The most of patients presents fever, dyspnea and cough as a typical viral infection, others show peculiar clinical and laboratory signs, such as anosmia, ageusia and thrombocytopenia. We here describe a severe coronavirus disease 2019 patient (76-year old, male) that developed an immune thrombocytopenia and acquired pseudo-thrombocytopenia that were refractory to immunomodulators even after resolution of respiratory failure. The patient developed thrombocytopenia (platelets $88,000 / \mathrm{mm}^{3}$ ) that got worse the following day $\left(14,000 / \mathrm{mm}^{3}\right)$. We started 1 $\mathrm{mg} / \mathrm{kg}$ of methylprednisolone i.v. daily. Platelet count increased up to $209,000 / \mathrm{mm}^{3}$, in sodium citrate, but remained about $14,000 / \mathrm{mm}^{3}$ in EDTA 10 days after the beginning of methylprednisolone. The patient showed great improvement in respiratory parameters and radiological finding. About one week after he developed a thrombocytopenia up to $70,000 / \mathrm{mm}^{3}$. We did not modify the steroids dosage. Platelet count slowly began to increase and in about 10 days returned to normal values.
\end{abstract}

\section{Introduction}

Since December 2019, a novel coronavirus [designated severe acute respiratory syndrome-related coronavirus 2 (SARSCoV-2)] has spread worldwide diffusing a new disease that World Health Organization (WHO) called coronavirus disease 2019 (COVID-19). ${ }^{1}$ This disease has peculiar characteristics both respiratory and clinical. As to respiratory findings, patients often present the so-called silent hypoxemia where they refer mild respiratory symptoms despite a severe saturation reduction and a severe hypoxia at arterial blood gases (ABG) analysis. This is due to a diffuse interstitial pneumonia that cause a severe alveolar impairment but preserve the lung compliance, so the patient, in the early stages, can compensate the hypoxia increasing the respiratory effort. ${ }^{2}$ This is evident as a respiratory alkalosis at $\mathrm{ABG}$ and has been called by some authors as type 1 pneumonia. On the other hand, sometimes patients present a type 2 pneumonia that shows opposite characteristics, so low lung compliance and high lung weight that recalls a typical acute respiratory distress syndrome (ARDS). ${ }^{3}$ Moreover, some authors suggest that type 1 pneumonia could evolve to a type 2 in some circumstances causing a greater respiratory effort and perhaps a worse outcome. As to clinical characteristics, there is great variability in presentation and laboratory findings. Even though the most of patients presents fever, dyspnea, fatigue and cough as a typical viral infection, ${ }^{4}$ some others show peculiar clinical and laboratory signs, such as anosmia, ageusia and thrombocytopenia. Thrombocytopenia, particularly, can be evident up to $57 \%$ of patients among severe cases. ${ }^{5}$ Its physiopathology includes: reduced production, increased intravascular destruction and higher consumption. ${ }^{6}$ The cytokine storm, described in SARS-CoV-2 infection, could be responsible of all these mechanisms and, moreover, can cause the lung damage that leads to the respiratory failure. ${ }^{7}$ Although the greatest part of COVID-19 thrombocytopenia is mild and rarely complicated by hemorrhages, some patients present a severe form. Some authors, indeed, recently reported a case of a severe COVID-19 related thrombocytopenia efficaciously treated with immunomodulators (steroid and immunoglobulins) and eltrombopag. ${ }^{8}$ We here describe a severe COVID-19 patient that developed an ITP and acquired pseudo-thrombocytopenia that were refractory to immunomodulators even after resolution of respiratory failure. An informed consent was obtained to write this case report.

\section{Case Report}

We describe a case report of a patient (76 years old, male) admitted in the emergency department in April 2020 for severe acute respiratory failure and fever. He presented a past history of chronic obstructive pulmonary disease in first class of GOLD classification, coronary artery disease in conservative treatment, hypertension and mild chronic kidney disease. At admission, the patient was afebrile, presented severe dyspnea, tachypnea and a good hemodynamic compensation (blood pressure 150/80 $\mathrm{mmHg}$, heart rate $80 \mathrm{bpm})$. He arrived in reservoir mask as oxygen supplementation
Correspondence: Mirko Di Capua,

Emergency Department, ASST Lodi, Largo donatori del sangue 1, 26900 Lodi, Italy. Email: mirdica@hotmail.com

Key words: COVID-19; immune thrombocytopenia; respiratory failure.

Acknowledgments: we wish to thank all the Emergency Department staff for all the efforts during the SARS-CoV-2 pandemic.

Contributions: all authors managed the patient during the admission. MDC wrote the article and all authors approved the final version before submission.

Conflict of interests: the authors declare no potential conflict of interests.

Informed consent: an informed consent was obtained to write this case report.

Received for publication: 15 January 2021. Revision received: 22 April 2021.

Accepted for publication: 19 March 2021

This work is licensed under a Creative Commons Attribution-NonCommercial 4.0 International License (CC BY-NC 4.0).

${ }^{\circ}$ Copyright: the Author(s), 2021

Licensee PAGEPress, Italy

Geriatric Care 2021; 7:9614

doi:10.4081/gc.2021.9614

and was evident, at $\mathrm{ABG}$, hypoxemia with an acute respiratory alkalosis and hyperlactatemia due to the respiratory effort $(\mathrm{pH}$ 7.61, $\mathrm{pCO}_{2} 16.4 \mathrm{mmHg}, \mathrm{pO}_{2} 63 \mathrm{mmHg}$ in $\mathrm{FiO}_{2} 80 \%$, lactate $3.71 \mathrm{mmol} / \mathrm{L}, \mathrm{p} / \mathrm{F} 78$ $\mathrm{mmHg}$ ). We immediately started a treatment with helmet continuous positive airway pressure with $10 \mathrm{cmH}_{2} \mathrm{O}$ of positive end expiratory pressure and $\mathrm{FiO}_{2}$ of $60 \%$. The physical examination showed only some inspiratory crackles, for the rest was unremarkable. As to laboratory findings, he showed mild hyperglycemia and mild worsening in creatinine levels (182 and 1.32 $\mathrm{mg} / \mathrm{dL}$ respectively), lactic dehydrogenase was $489 \mathrm{IU} / \mathrm{L}$, normal values (n.v.) range from 5 to $248 \mathrm{IU} / \mathrm{L}$, and C-reactive protein was $31.7 \mathrm{mg} / \mathrm{L}$ (n.v. 0-7.5). Ferritin, at presentation, was $1500 \mathrm{ng} / \mathrm{mL}$ (n.v. 24-336), ddimer levels were $1590 \mathrm{mg} / \mathrm{L}$ (n.v. 50-500), while transaminase, procalcitonin and electrolytes were in normal ranges. The nose swab confirmed a SARS-CoV-2 infection. A complete blood count (CBC) showed a mild anemia (hemoglobin $10.9 \mathrm{gr} / \mathrm{dL}$ ), but stable compared to previous $\mathrm{CBCs}$, a mild lymphopenia (lymphocytes $670 / \mathrm{mm}^{3}$; n.v. $900-$ $5200)$ and normal platelets $\left(167,000 / \mathrm{mm}^{3}\right)$, even if they were reduced compared to a 
previous CBC $\left(293,000 / \mathrm{mm}^{3}\right)$. A point of care ultrasound showed a diffuse patchy distribution of interstitial artifactual signs with some pleural consolidations compatible with a COVID-19 interstitial pneumonia, a small and reactive inferior vena cava and normal heart dimension with a mid-range ejection fraction impairment (about 45\%). We started a drug therapy, as suggested by our internal protocol, with enoxaparin 4000 UI qd, hydroxychloroquine $200 \mathrm{mg}$ qid the first day and then bid for other 6 days, azithromycin $500 \mathrm{mg}$ qd for 5 days. The day after admission the patient developed thrombocytopenia (platelets $88,000 / \mathrm{mm}^{3}$ ) that got worse the following day $\left(14,000 / \mathrm{mm}^{3}\right)$. The analyzer flagged the presence of platelet clumps. We did not find any sign of hemolysis and a peripheral blood smear did not show schistocytes but confirmed thrombocytopenia and some aggregates. We performed a platelet count with sodium citrate that confirmed the thrombocytopenia but only mild $\left(69,000 / \mathrm{mm}^{3}\right)$ and did not show platelet clumps in sodium citrate. Coagulation values remained into normal limits. As the patient received heparin also 40 days before, we researched anti PF4 antibodies that resulted negative, while antiplatelet antibodies (specifically anti glycoprotein IIb-IIIa) resulted positive. We started $1 \mathrm{mg} / \mathrm{kg}$ of methylprednisolone i.v. daily. We suspended hydroxychloroquine. Five days after admission, the patient still presented a severe respiratory failure, as he needed high flow nasal cannulas (HFNC) at $\mathrm{FiO}_{2} 60 \%$ and $60 \mathrm{~L} / \mathrm{min}$ of flow to achieve a saturation of $92 \%$ and the lung ultrasound showed an initial evolution to an ARDS pattern. Platelet count was still $13,000 / \mathrm{mm}^{3}$ in EDTA and $110,000 / \mathrm{mm}^{3}$ in sodium citrate. We decided to administrate tocilizumab as the platelet count in sodium citrate showed a partial improvement and the patient was in the inflammatory phase of the disease. We administered $8 \mathrm{mg} / \mathrm{kg}$ of tocilizumab twice at 12 hours of distance from the first dose and maintained the methylprednisolone. In the following days the patient presented progressive improvement of respiratory failure and platelet count. About 3 days after tocilizumab administration, the patient was completely weaned from HFNC and achieved a saturation of $96 \%$ with only $2 \mathrm{~L}$ per minute of oxygen. Platelet count increased up to $209,000 / \mathrm{mm}^{3}$, in sodium citrate, but remained about $14,000 / \mathrm{mm}^{3}$ in EDTA 10 days after the beginning of methylprednisolone and 6 days after tocilizumab administration. The patient showed great improvement in respiratory parameters and radiological finding as shown in Figure 1. He was transferred to a respiratory rehabilitation ward and continued steroids even if at a lower dosage (methylprednisolone $20 \mathrm{mg} /$ daily). About one week after he developed a thrombocytopenia relapse with a reduction in platelet count of $143,000 / \mathrm{mm}^{3}$ in sodium citrate

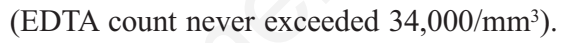

Two days after the thrombocytopenia became $111,000 / \mathrm{mm}^{3}$ and then $70,000 / \mathrm{mm}^{3}$ in sodium citrate. We did not modify the steroids dosage as the patient had an iatrogenic diabetes with poor pharmacological control and he was asymptomatic as to hemorrhagic events. Platelet count slowly began to increase and in about 10 days returned to normal values both the sodium citrate and the EDTA count, showing a resolution of the acquired pseudo-thrombocytopenia too (Figure 2).

\section{Discussion}

SARS-CoV-2 infection leads to a disease with multiple organ and laboratory involvement. Even if many aspects of COVID-19 are becoming clearer - as research goes on - many others are still unknown. The greatest part of present literature has investigated the acute phase of SARS-CoV-2 infection but poor is known about the post-acute phase. Scarce data are available of post infection period and we are not aware of the possible sequelae. Some authors suggest that the greatest part of hematological and hemostatic alterations (and sometimes the worse outcome) are due to the hyper inflammatory status (sometimes called cytokine storm) related to SARS-CoV-2 infection. ${ }^{7}$ On the other hand, it is still unknown how long the effects of
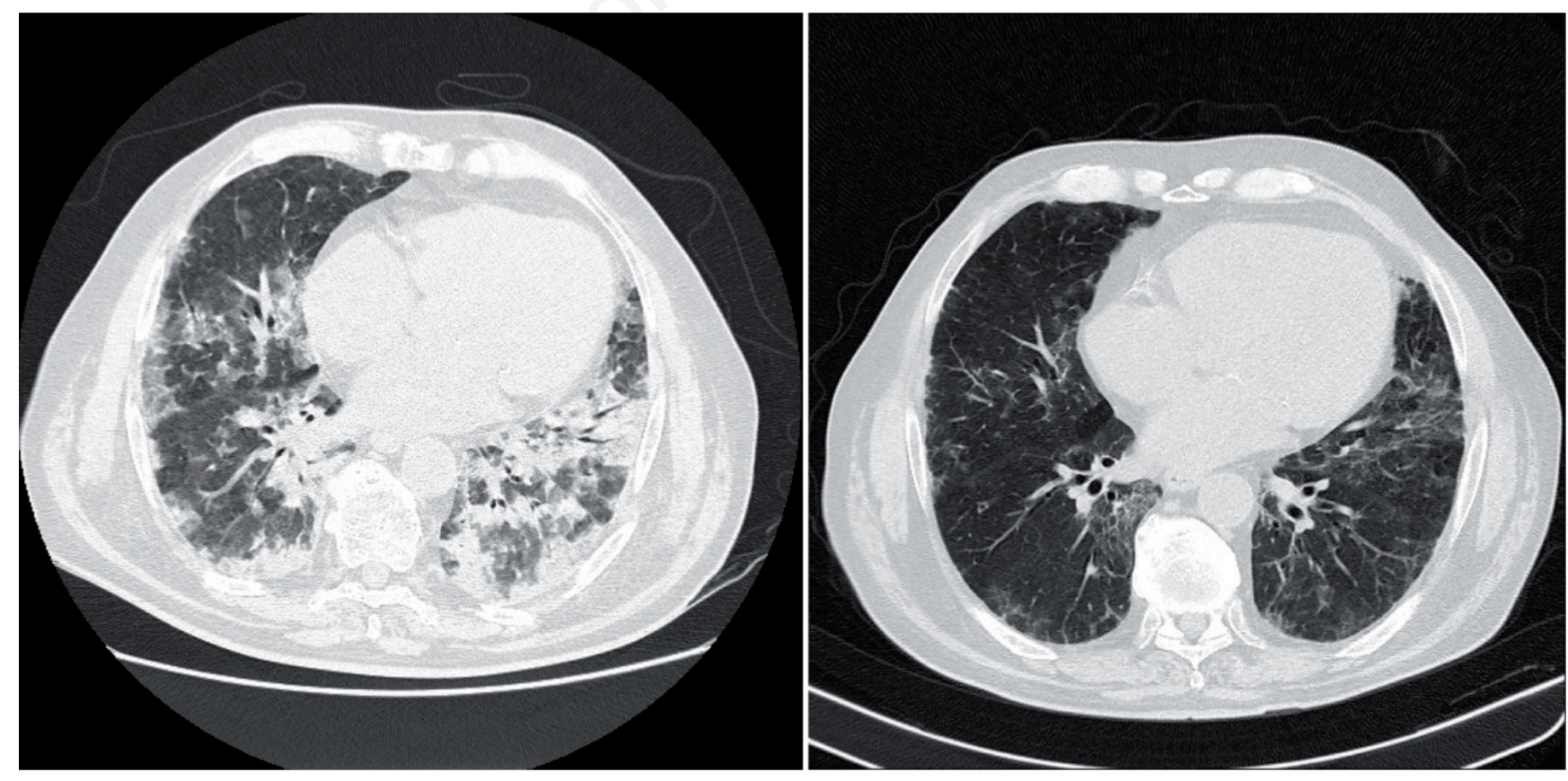

Figure 1. In this figure we present a high resolution computed tomography (HRCT) of the chest at day 0 (left), showing a severe interstitial COVID-19 pneumonia with multiple and diffuse consolidations. On the right the same HRCT scan after 11 days of therapy. 


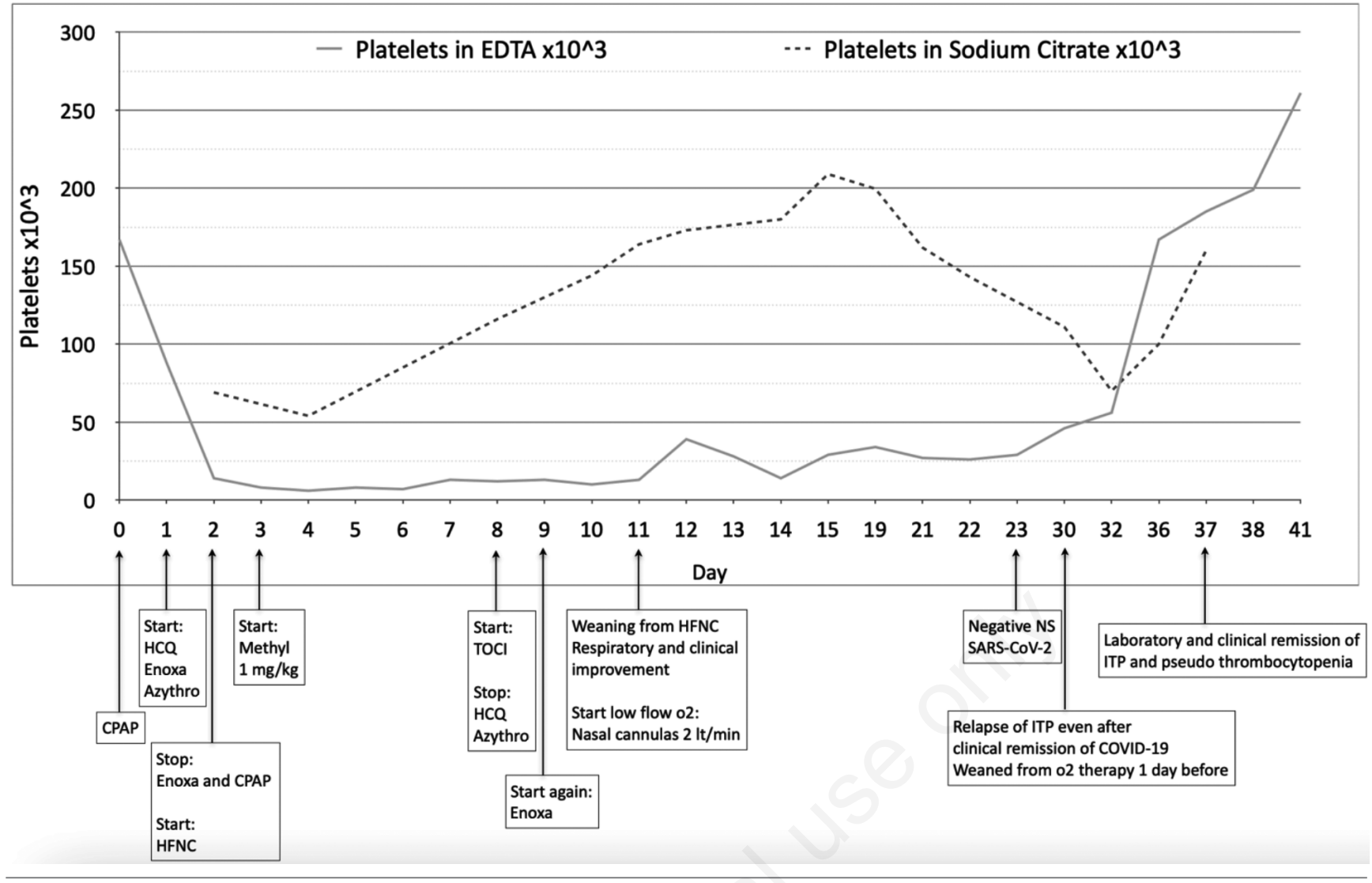

Figure 2. This figure shows platelet count in EDTA and sodium citrate during the whole admission. It put in evidence the relapse of immune thrombocytopenia (ITP) even after clinical remission from COVID-19 and a nasal swab (NS) negative for SARS-CoV-2 detection. The continuous line indicates the platelet count in EDTA, the dashed line indicates the platelet measurement in sodium citrate. CPAP stands for continuous positive airway pressure, HCQ is hydroxychloroquine ( $200 \mathrm{mg}$ qid the first day, then bid), enoxa is enoxaparine (4000 UI qd), azythro stands for azythromicine (500 mg qd), methyl is methylprednisolone (1 mg/kg/die), TOCI is tocilizumab $(8 \mathrm{mg} / \mathrm{kg}$ in two administrations at 12 hours of distance), HFNC stands for high flow nasal cannulas.

the cytokine storm can persist and so how long the patients are at higher risk of complications. The present case report suggests that some COVID-19 complications, particularly hematological and hemostatic ones, could continue beyond respiratory and clinical resolution of the disease. As shown in Figure 1, the patient had almost a completely radiological resolution of the SARSCoV-2 pneumonia but they still presented thrombocytopenia. Moreover, the nose swab has become negative much earlier suggesting a complete virus eradication without a concrete reduction of complication incidence. The pandemic spread of SARS-CoV-2 has put to the test the whole healthcare system and its real implication on the health care system are still to be evaluated, ${ }^{9}$ as many patients will probably develop some complication at distance from acute infection. Our case report argues for an adequate monitoring of the patients, particularly the ones at higher risk, as they could develop some complications even after a complete clinical resolution of the disease and a negative nose swab.

\section{Conclusions}

Even if thrombocytopenia is common among severe COVID-19 patients, it is usually mild and asymptomatic. This case report describes a severe immune thrombocytopenia and a rare case of acquired pseudo thrombocytopenia that, to our knowledge, has not yet been described in COVID-19 patients. Moreover, acquired pseudo thrombocytopenia lasted and the ITP relapsed even after clinical remission and a negative nose swab. This suggests that patients could keep a high risk of COVID-19 complications even after clinical remission and probably after patient dismission, perhaps due to the severe inflammatory state and the cytokine storm typical of these patients. We are aware that clinical research studies are needed to confirm our report; should this be the case, it is reasonable to implement a clinical follow-up after disease resolution, particularly for fragile geriatric patients that are at higher risk of morbidity and mortality related to SARSCoV-2 infection.

\section{References}

1. Zhu N, Zhang D, Wang W, et al. A novel coronavirus from patients with pneumonia in China, 2019. N Engl J Med 2020;382:727-33.

2. Gattinoni L, Coppola S, Cressoni M, et al. COVID-19 does not lead to a "typical" acute respiratory distress syndrome. Am J Respir Crit Care Med 2020. [Epub ahead of print].

3. Gattinoni L, Chiumello D, Rossi S. COVID-19 pneumonia: ARDS or not? Crit Care 2020;24:154.

4. Wang D, Hu B, Hu C, et al. Clinical characteristics of 138 hospitalized patients with 2019 novel coronavirusinfected pneumonia in Wuhan, China. JAMA 2020. [Epub ahead of print].

5. Guan WJ, Ni ZY, Hu Y, et al. Clinical characteristics of coronavirus disease 2019 in China. N Engl J Med. 2020;382:1708-20.

6. Panyang Xu, Qi Zhou, Jiancheng Xu. Mechanism of thrombocytopenia in 
COVID-19 patients. Ann Hematol 2020;99:1205-08.

7. Fajgenbaum DC, June CH. Cytokine storm. N Engl J Med 2020;383: 2255-73.
8. Zulfiqar A-A, Lorenzo-Villalba N, Hassler P, Andrès E. Immune thrombocytopenic purpura in a patient with COVID-19. N Engl J Med 2020; 382:e43.
9. Phelan AL, Katz R, Gostin LO. The novel coronavirus originating in Wuhan, China: challenges for global health governance. JAMA 2020. [Epub ahead of print]. 